\title{
Clasificación de género utilizando medidas antropométricas, análisis de texturas y la arquitectura ANFIS
}

\author{
Cosijopii García García, Nayeli Joaquinita Meléndez Acosta \\ Universidad del Istmo Campus Ixtepec, Ciudad Ixtepec, Oaxaca, \\ México \\ \{cosijopiigarcia, nayelimelendez\}@gmail.com
}

\begin{abstract}
Resumen. La automatización del proceso de clasificación de género está justificado por diversas aplicaciones que juegan un rol muy importante en la interacción entre los humanos y la computadora como: motores de búsqueda, recolección de datos demográficos y como parte de frameworks biométricos y psicológicos. En el presente artículo se diseña un método de clasificación de género utilizando imágenes faciales y la arquitectura ANFIS como clasificador, la cual nos ofrece las ventajas de la lógica difusa y las redes neuronales. La metodología propuesta nos llevará por varias fases, en las cuales se usarán diferentes métodos para la extracción de las características del rostro, como son ojos, frente, nariz, boca, barbilla, etc., así como el algoritmo LBP (Local Binary Pattern) que ayudan en la extracción de texturas y el análisis antropométrico. Se realizaron pruebas utilizando 428 imágenes los cuales fueron introducidos al clasificador, y se obtuvo un porcentaje de clasificación del $86.44 \%$.
\end{abstract}

Palabras clave: ANFIS, imágenes faciales, clasificación, LBP, clasificación de género, análisis de texturas, lógica difusa, FCM.

\section{Gender Classification \\ Using Anthropometric Measurements, Texture Analysis and ANFIS Architecture}

\begin{abstract}
The automation of the gender classification process is justified by several applications that play a very important role in the interaction between humans and the computer such as: search engines, demographic data collection and as part of biometric and psychological frameworks. This paper designs a method of gender classification using facial images and the ANFIS architecture as a classifier, which offers us the advantages of diffuse logic and neural networks. The proposed methodology will take us through several phases, in which different methods will be used for the extraction of facial characteristics, such as eyes, forehead, nose, mouth, chin, etc., as well as the LBP algorithm that help in the extraction of textures and anthropometric analysis. Tests were
\end{abstract}


performed using 428 images which were introduced to the classifier, and a grading percentage of $86.44 \%$ was obtained.

Keywords: ANFIS, facial images, classification, LBP, gender classification, texture analysis, fuzzy logic, FCM.

\section{Introducción}

El rostro humano nos provee de la forma más rápida y directa, la posibilidad de evaluar implícitamente información social crítica, por ejemplo, el rostro humano nos puede dar información acerca de la raza, el género, la edad, expresiones e identidad, los cuales son de utilidad para ayudar al proceso de toma de decisiones [1].

La investigación del comportamiento psicológico muestra, que el encuentro con un nuevo individuo frente a un estimuló del rostro humano activa tres evaluaciones neuronales primitivas, la raza, el género y la edad, estos tienen consecuentes para el perceptor y el percibido [1]. Por lo tanto este problema ha inspirado a investigadores que se han dedicado al análisis facial, con énfasis especial en la identificación del rostro, extracción de características, clasificación de género, edad y expresiones [2].

La identificación del género está justificada por diversas aplicaciones en diferentes áreas como: La interacción humano computadora (HCI) y la seguridad [3].

En la indexación de bases de datos de imágenes, optimizando las búsquedas y mejorando el tiempo de consulta, así como reduciendo los espacios de búsqueda en bases de datos muy grandes, también como el ordenamiento de fotos digitales [4].

También se puede extrapolar a áreas como el marketing digital mostrando publicidad a un tipo de género $[4,5]$.

Tiene aplicaciones en la autenticación de usuarios y como parte de sistemas de seguridad biométricos [6]. Y en la vigilancia y re identificación de sujetos utilizando cámaras CCTV y Soft Biometrics [6, 7].

Este artículo está organizado por secciones, la primera explica el trabajo relacionado en la clasificación de género, así como los materiales y la metodología que se utilizó, la sección dos explica como la imagen inicialmente es normalizada y pre procesada para la extracción de características, la sección tres explica cómo se encontraron los puntos del rostro y como se obtuvieron las medidas antropométricas del rostro, así como el uso de LBP para el análisis de texturas, la sección cuatro nos muestra cómo se prepararon los datos y el uso de ANFIS para la clasificación así como el uso del algoritmo FCM para la creación de un modelo difuso el cual ANFIS optimizó, en las secciones cinco y seis nos muestran los resultado de la clasificación y las validaciones que se realizaron al modelo, por ultimo las conclusiones a las que se llegaron con el modelo realizado y las referencias.

\subsection{Trabajo relacionado}

Una revisión de los métodos de clasificación de género es dada por [8] y más recientemente también por [6]. Algunos puntos relevantes son los siguientes: uno de 
Clasificación de género utilizando medidas antropométricas, análisis de texturas y la arquitectura ...

los primeros métodos utilizados para la clasificación de género fueron las redes neuronales, esta red fue entrenada con un conjunto de imágenes faciales frontales pequeñas, este pequeño clasificador fue llamado SEXNET [9].

En [10] se utiliza la combinación de la estructura en 3D del rostro humano extraído por un escáner laser y las intensidades de la imagen, esto para clasificar el género. Los clasificadores SVM fueron utilizados por [11] usando directamente las intensidades de la imagen, algo raro en el uso de SVM's [12].

En [13] usa AdaBoost para la clasificación de género aplicando de igual manera las intensidades de la imagen. En años más recientes autores como [3] han usado Webers Local texture descriptors para el reconocimiento del género, utilizando la base de imágenes faciales FERET [14].

Los autores [15] proponen un método usando redes neuronales para la clasificación de grupos de edad y género, el cual utiliza 4 grupos de edad, su clasificación está basada en la geometría del rostro dependiendo del género, y en el análisis de textura de la piel, su metodología propuesta se divide en tres pasos principales : preprocesamiento , extracción de características y clasificación.

En [7] proponen un método utilizando un descriptor Zernike moments, combinando estos datos con lógica difusa, construyeron un sistema de inferencia difuso. En [12] proponen un método de clasificación utilizando redes convolucionales, entrenan la CNN (Convolutional Neural Network) con imágenes de la base de datos Adience benchmark for age and gender classification, estos proponen pasar la imagen por varios filtros a través de la red, cada filtro aplica una operación de convolución y la pasa a la siguiente capa de la red.

La arquitectura ANFIS ha sido también utilizada en la clasificación de género, mediante diferentes tipos de métodos como la utilización de la voz [16] y de imágenes de huellas dactilares [17].

\subsection{Materiales y métodos}

Para el desarrollo de esta investigación se utilizaron imágenes faciales de dos bases de datos, FERET [14] y PAL [18] ya que contienen imágenes de rostros frontales y alineados. Las bases de datos se unieron para formar un total de 1437 imágenes, siendo 704 imágenes faciales femeninas y 733 imágenes faciales masculinas, la base de datos FERET está compuesta por imágenes de tamaño $640 \times 480$ píxeles y la base de datos PAL de $192 \times 128$ píxeles. Se utilizó la herramienta matemática MATLAB ya que nos provee de herramientas especializadas para el trabajo con redes neuro-difusas en especial la arquitectura ANFIS.

La metodología de desarrollo que se utilizó está basada en los siguientes autores [15], [5] y [2], los cuales proponen una metodología para el problema de la clasificación de edad y género utilizando comúnmente las redes neuronales, en este caso se usó en la fase de clasificación la arquitectura ANFIS [19], la cual es un tipo de red neuro difusa que utiliza las técnicas de lógica difusa y redes neuronales, por lo tanto de la combinación de estas técnicas se espera una herramienta poderosa [20].

A continuación, se muestran las fases y el diagrama donde se observan las diferentes etapas de la metodología que se utilizaron en esta investigación, la metodología está 
dividida en 3 secciones principales: preprocesamiento, extracción de características y clasificación (véase Fig. 1)

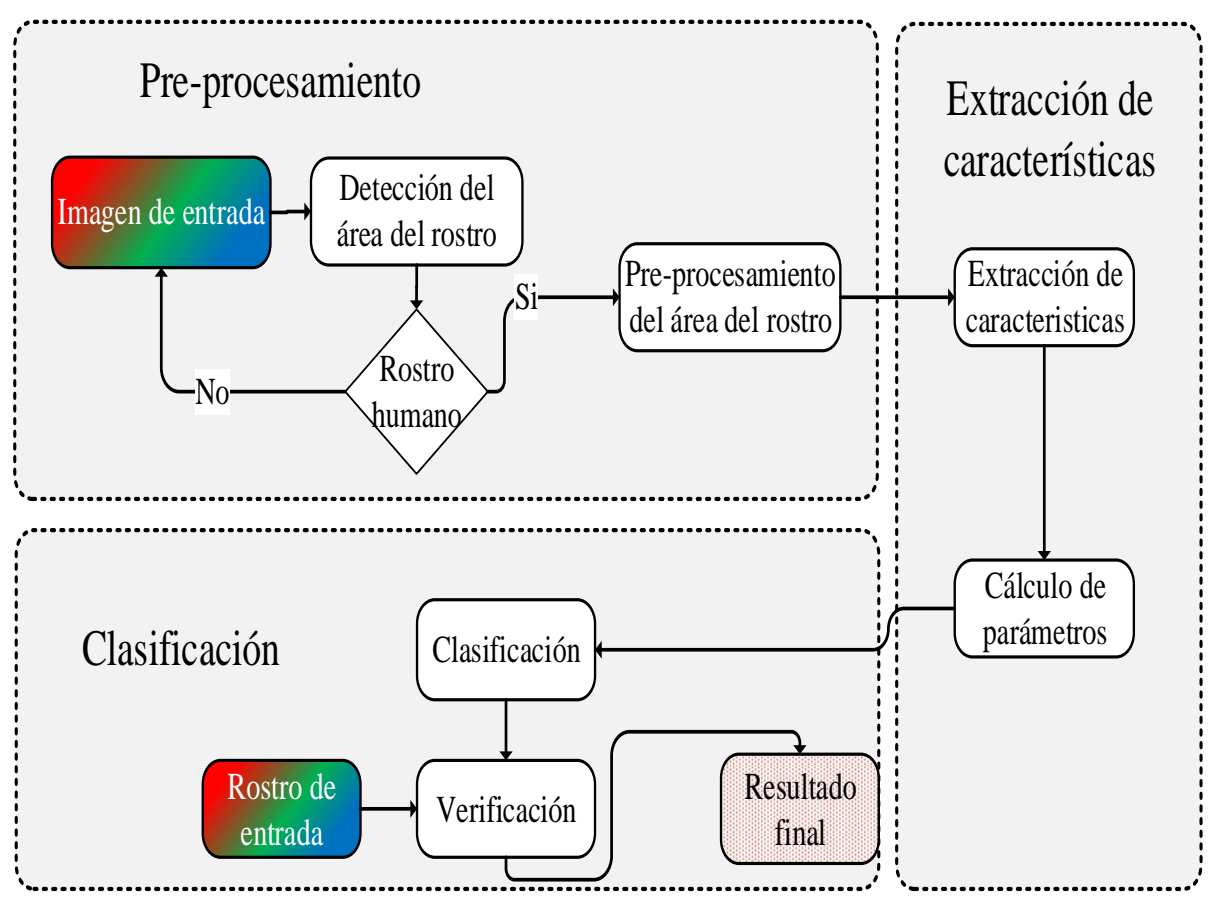

Fig. 1. Etapas de la metodología propuesta para la resolución de la clasificación de género.

\section{Preprocesamiento del rostro}

La imagen del rostro humano fue normalizada en tamaño, color y aspecto, véase Fig. 2 (a) y (b), esto para que el rostro sea fácilmente encontrado, haciendo que el paso (a) y (b) funcionen con cualquier imagen de tamaño diferente, para esto se utilizó un umbral en el que si el tamaño de la imagen es mayor que $640 \times 480$ píxeles, esta se reduce a la mitad y si es menor que esta resolución, se duplique el tamaño de la imagen utilizando un algoritmo de interpolación bilineal, esto para reducir la perdida de información, posteriormente se busca si la imagen contiene una rostro humano utilizando el algoritmo de Viola-Jones [21] véase Fig. 2 (c), para luego realizar el corte del área del rostro de manera automática y posteriormente convertir la imagen a un tamaño predeterminado de $100 \times 100$ píxeles, esto con el propósito de normalizar la imagen recortada sin importar el tamaño de la imagen de entrada, esta imagen es la que se utilizará en todos los procesos siguientes, véase Fig. 2 (d). 
Clasificación de género utilizando medidas antropométricas, análisis de texturas y la arquitectura ...

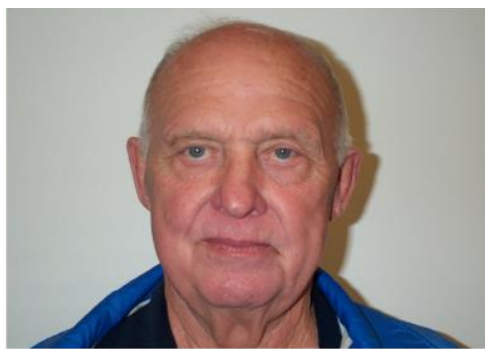

a) Imagen original $(640 \times 480)$

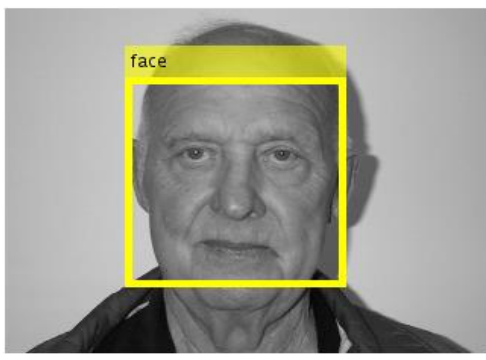

c) Localización del área del rostro

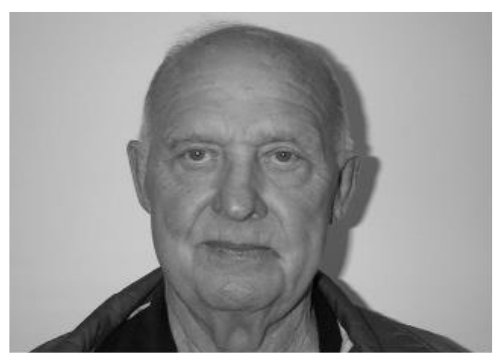

b) Imagen a escala de grises $(320$ x 240$)$

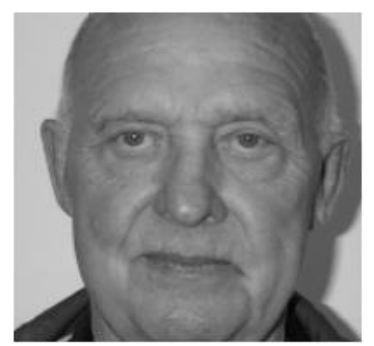

d) Área del rostro recortada (100 x 100)

Fig. 2. Preprocesamiento de la imagen facial para encontrar el área del rostro.

\section{Extracción de características}

La imagen normalizada y convertida a escala de grises es utilizada para ubicar diferentes puntos en el rostro humano, los puntos fueron escogidos siguiendo el estudio de la antropometría del rostro según [22]. A estos le añadimos algunos puntos que son utilizados en la antropometría del rostro de [2], el conjunto completo de puntos se muestra en la Fig. 3.

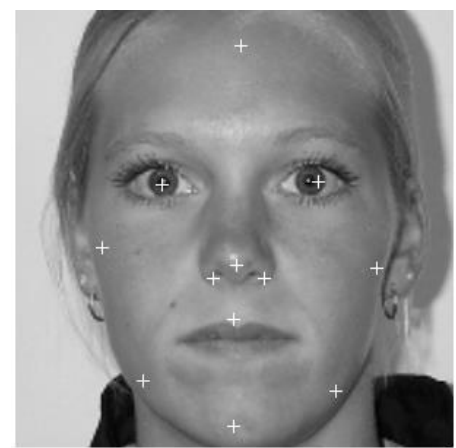

Fig. 3. Puntos del rostro humano seleccionados

Para ubicar los puntos se usaron dos técnicas diferentes, en la ubicación de los ojos se utilizó un clasificador Viola-Jones, que ya ha sido entrenado con imágenes que contengan a ambos ojos como se ve en la Fig. 4. 


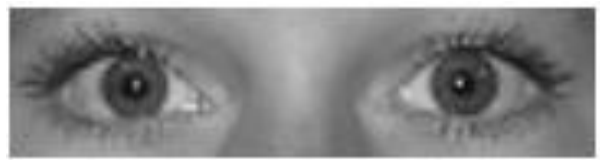

Fig. 4. Par de ojos recortados de la imagen facial.

Teniendo la imagen de los ojos se procede a realizar un recorte de cada ojo, estos recortes se hacen de manera automática, siendo el tamaño de la imagen recorta dependiendo del tamaño del área de los ojos, de esta forma se tienen dos imágenes nuevas en las cuales se buscará el iris, siendo este el centro de los ojos. Como primer paso se realizó una ecualización del histograma de cada imagen, posteriormente cada imagen es reducida de todos los lados hacia el centro 35 píxeles, se escogió esta cantidad porque proporciona los mejores resultados, esto se realizó en cada imagen de manera automática, para eliminar el posible ruido alrededor de nuestro objetivo, el iris (véase Fig. 5).
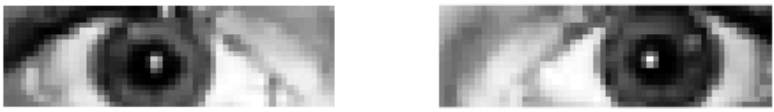

Fig. 5. Ecualización del histograma de ambos ojos, así como el recorte para centrar el iris de cada ojo.

Utilizando estas dos imágenes se procede a encontrar el mínimo local que existe en cada imagen, este siempre tiende a ser el iris por la poca intensidad que contiene los píxeles cercanos [22] (véase en la Fig. 6).

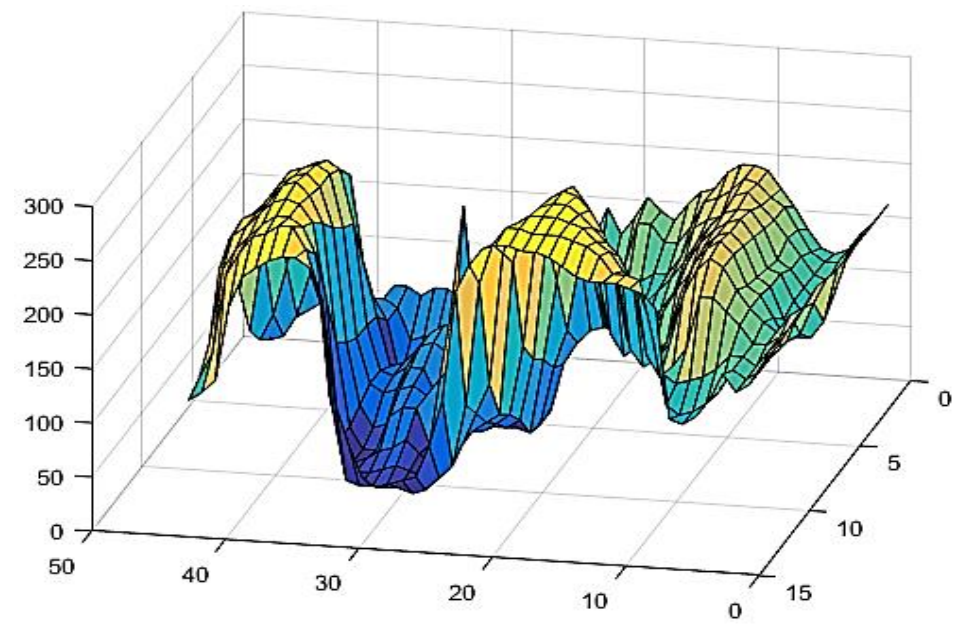

Fig. 6. Representación en 3D de una imagen del ojo derecho se puede observar que las partes más oscuras están ubicadas en el iris del ojo, las partes amarillas corresponden a la esclerótica del ojo. 
Clasificación de género utilizando medidas antropométricas, análisis de texturas y la arquitectura ...

Teniendo las coordenadas de los ojos se procede a ubicar el centro de la frente, utilizando la distancia que existen entre los ojos mediante la siguiente ecuación:

$$
D_{\text {eye }}=\sqrt{\left(x_{1}-y_{1}\right)^{2}+\left(x_{2}-y_{2}\right)^{2}} .
$$

Posteriormente se utilizó una analogía del triángulo equilátero, usando la cúspide del triángulo como el centro de la frente (véase Fig. 7), la altura del triángulo es encontrado utilizando la siguiente ecuación:

$$
h=\frac{D_{\text {eye }} \sqrt{3}}{2} .
$$

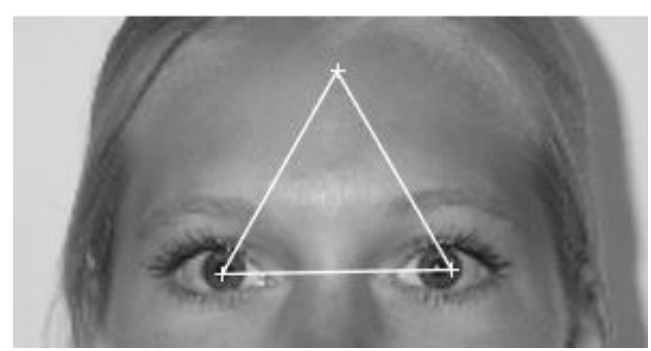

Fig. 7. La distancia que existe entre los ojos, es utilizada como base de un triángulo equilátero y de esta manera se encuentra en la cúspide el centro de la frente.

Para ubicar el resto de los puntos mostrados en la Fig. 3 se utilizó el proyecto OpenFace [23], el cual es un framework que nos proporciona la posibilidad de encontrar en el rostro hasta 68 características, sin embargo, ni la frente, ni el iris de cada ojo son parte de ellas. El proceso por el cual OpenFace encuentra las características es el siguiente: utiliza un preprocesamiento y redes neuronales para obtener una representación de bajo nivel del rostro, el preprocesamiento se divide en dos partes: una transformación para alinear los ojos y la nariz, más la detección de las 68 marcas en el rostro utilizando la librería dlib's face landmark detector, posteriormente la imagen es recortada e introducida a una red neuronal la cual fue entrenada con una base de datos privada llamada FaceNet, esta base de datos contiene de 100 a 200 millones de imágenes [23].

Tabla 1. Medición entre puntos del rostro.

\begin{tabular}{cc}
\hline Característica & Medidas \\
\hline F1 & Distancia de P9 a P11 \\
F2 & Distancia de P10 a P12 \\
F3 & Distancia de P3 a P7 \\
F4 & Distancia de P1 a P12 + Distancia de P12 a P2 \\
F5 & Distancia de P8 a P12 \\
F6 & Distancia de P4 a P5 + Distancia de P5 a P6 \\
F7 & Distancia de P3 a P4+ Distancia de P4 a P5 + Distancia de P5 a P6 + \\
& Distancia de P6 a P7 \\
\hline
\end{tabular}




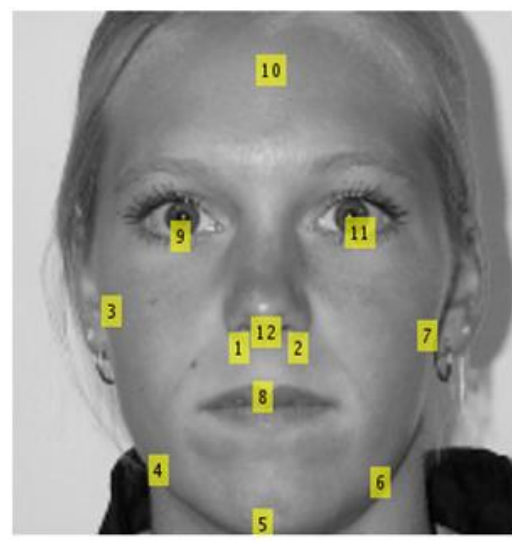

a) Puntos del rostro numerados

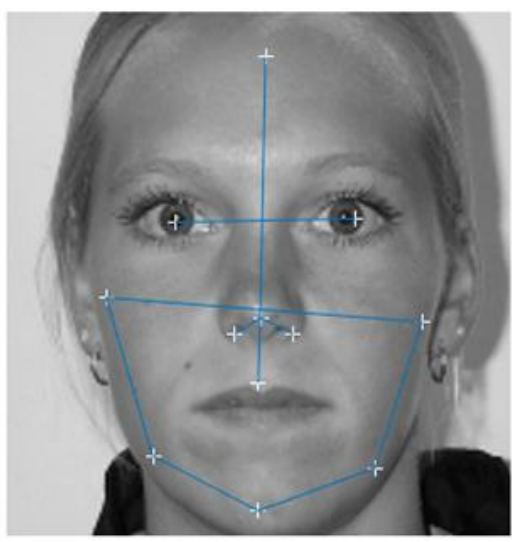

b) Medición entre puntos del rostro

Fig. 8. Puntos del rostro numerados y líneas que definen las distancias entre los puntos.

El ubicar los puntos fue necesario para establecer 7 características las cuales son medidas entre los puntos encontrados en la imagen, las medidas fueron inspiradas en [15], véase Fig. 8 (b), a continuación, en la Tabla 1, se muestran las características y la forma en la que fueron extraídas según la Fig. 8 (a).

Después de extraer las características utilizando las medidas antropométricas, se procede a utilizar LBP, el cual analiza las texturas de la imagen, esto se realiza separando la información de los patrones y del contraste, al hacer esto se obtienen los cambios invariantes en las imágenes a escala de grises [24]. LBP asigna un valor a cada píxel comparándolo con sus píxeles vecinos [25]. El operador LBP es definido como:

$$
L P B_{P, R}\left(x_{c}, y_{c}\right)=\sum_{p=0}^{P-1} s\left(g_{p}-g_{c}\right) 2^{p} \quad, \quad s(z)= \begin{cases}1, & z \geq 0 \\ 0, & z<0 .\end{cases}
$$

Donde $g_{c}=I(x, y)$ es el píxel central y $g_{p}=I\left(x_{p}, y_{p}\right), \quad p=0, \ldots, P-1$, es la intensidad de gris de la vecindad $P$ y $R$ del radio alrededor de los puntos $(x, y)$, los puntos $x_{p}$ e $y_{p}$ son calculados de la siguiente manera: $y_{p}=y-R \sin (2 \pi p / P) \quad \mathrm{y}$ $x_{p}=x+R \cos (2 \pi p / P)$.

Los patrones producidos por el operador $\operatorname{LPB}(p, r)$ describen la textura de la imagen, estos son llamados patrones uniformes, los cuales contienen al menos dos transiciones en el bit 0 o 1 y viceversa, se observó que la mayor parte de la información de textura está contenida en los patrones uniformes. Etiquetar los patrones que tienen más de 2 transiciones con una sola etiqueta produce un operador LBP, denominado $\operatorname{LBP}(P, R, u 2)$, que produce patrones sin perder demasiada información[26]. LBP dividió la imagen del rostro de $100 \times 100$ píxeles en 59 bloques para posteriormente procesar individualmente cada uno, esto nos devuelve el histograma de cada bloque, como resultado final los bloques se concatenan en un solo vector (véase Fig. 9). 


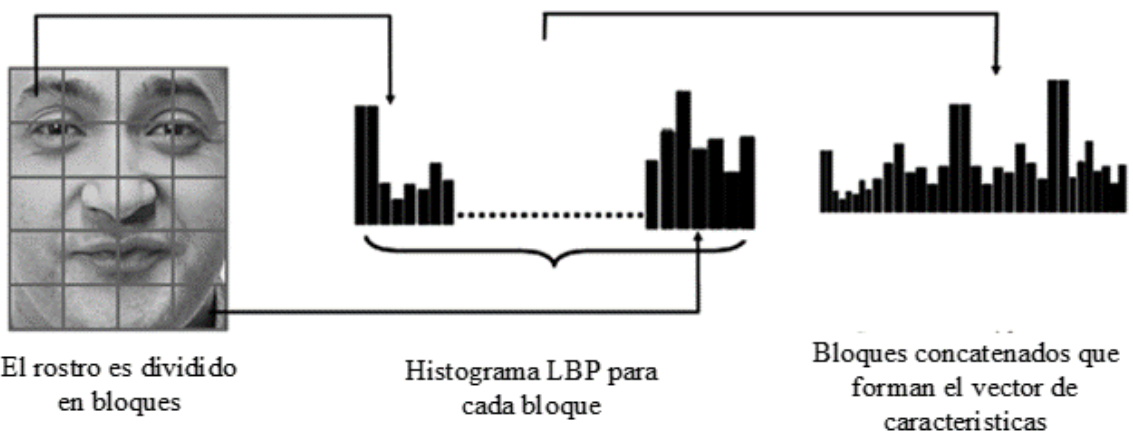

Fig. 9. Proceso por el cual LBP obtiene las características de textura de la imagen facial.

Este vector de 59 características se concatena al primer vector de 7 características teniendo así en total 66 características, el cual será utilizado en la fase de clasificación.

\section{Clasificación}

En esta fase se utilizó la arquitectura ANFIS (Adaptive Neuro Fuzzy Inference System), esta fue propuesta por R. Jang [19] en 1993. ANFIS puede lograr un mapeo altamente no lineal, muy superior a una red neuronal multicapa (MLP, Multilayer perceptron) y otros métodos lineales comunes de complejidad similar [27]. ANFIS es una red de cinco capas, véase Fig. 9; ésta requiere como entrada un sistema difuso tipo Sugeno el cual es optimizado mediante la modificación de los parámetros en el proceso de aprendizaje, tal proceso utiliza una combinación de mínimos cuadrados y el algoritmo Backpropagation, en otras palabras ANFIS utiliza las ventajas de la lógica difusa y las redes neuronales para ajustar sus parámetros y encontrar la solución óptima [20].

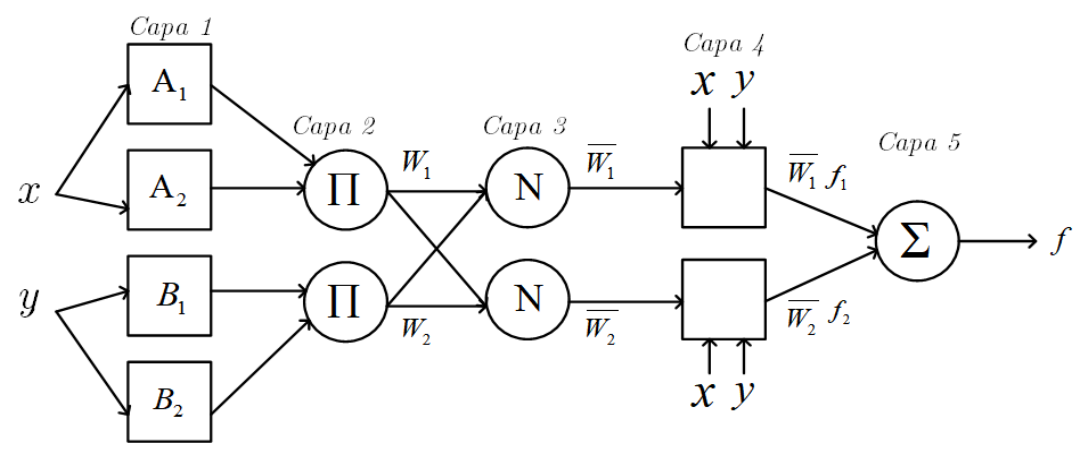

Fig. 10.Arquitectura de ANFIS equivalente a un modelo difuso tipo Sugeno.

Cada capa es especificada de la siguiente manera:

Capa 1. Cada nodo $i$ de esta capa es un nodo adaptativo con una función: 


$$
O_{1, i}=\mu_{A i}(x), i=1,2,
$$

donde $x$ o $y$ es la entrada del nodo $i$ y $A_{i}$ es una etiqueta lingüística asociada con tal nodo, en otras palabras $O_{i, 1}$ es el grado de pertenencia del conjunto difuso

Capa 2. Los nodos de esta capa son nodos fijos representados con el símbolo $\Pi$, la salida de los nodos se calcula como el producto de todas las señales de entrada:

$$
O_{2, i}=w_{i}=T\left(\mu_{A i}(x) \mu_{B i}(y)\right), i=1,2,
$$

donde la salida es calculada con una norma-T.

Capa 3. En esta capa cada nodo es etiquetado con $N$, el i-ésimo nodo calcula la relación de la intensidad del disparo de la i-ésima regla con respecto a la suma de las intensidades de disparo de todas las reglas:

$$
O_{3, i}=\overline{w_{i}}=\frac{w_{i}}{w_{1}+w_{2}}, i=1,2 .
$$

Capa 4. Cada nodo $i$ en esta capa es un nodo adaptado a la función:

$$
O_{4, i}=\overline{w_{i}} f_{i}=\overline{w_{i}}\left(p_{i} x+q_{i} y+r_{i}\right),
$$

donde $\bar{w}_{i}$ es la salida normalizada de la capa 3 y $\left\{p_{i} x+q_{i} y+r_{i}\right\}$ son el conjunto de parámetros que definen a los polinomios $Z$ en este nodo, los parámetros de este nodo son conocidos como parámetros del consecuente.

Capa 5. El único nodo que representa esta capa es denotado por $\Sigma$, el cual calcula la salida global como la suma de todas las señales de entrada:

$$
O_{5,1}=\sum_{i} \overline{w_{i}} f_{i}=\frac{\sum_{i} w_{i} f_{i}}{\sum_{i} w_{i}} .
$$

$\mathrm{Y}$ de esta forma se compone una red adaptativa equivalente a un sistema Sugeno de primer orden.

Para crear el sistema Sueno de primer orden que es utilizado por ANFIS, se recurrió al algoritmo Fuzzy C-Means el cual es un algoritmo iterativo que permite que cada dato pertenezca a un clúster con diferentes grados de pertenencia, la función objetivo se define de la siguiente manera:

$$
J_{m}=\sum_{i=1}^{D} \sum_{j=1}^{N} \mu_{i j}^{m}\left\|x_{i}-c_{j}\right\|^{2},
$$

donde $D$ es número de datos, $N$ el número de clúster, $m$ es la partición difusa la cual controla el grado de solapamiento difuso $m>1 . x_{i}$ es el i-ésimo dato, $c_{j}$ es el centro del j-ésimo clúster y por último $\mu_{i j}$ es el grado de pertenencia de $x_{i}$ en el j-ésimo clúster [28]. El algoritmo FCM fue configurado con 8 clústers iniciales y los datos introducidos fueron las 66 características de 1009 imágenes (véase Fig. 11), esto con la finalidad que el algoritmo nos proporcione un FIS (Fuzzy inference System) inicial el cual fue optimizado por ANFIS. 
Clasificación de género utilizando medidas antropométricas, análisis de texturas y la arquitectura ...

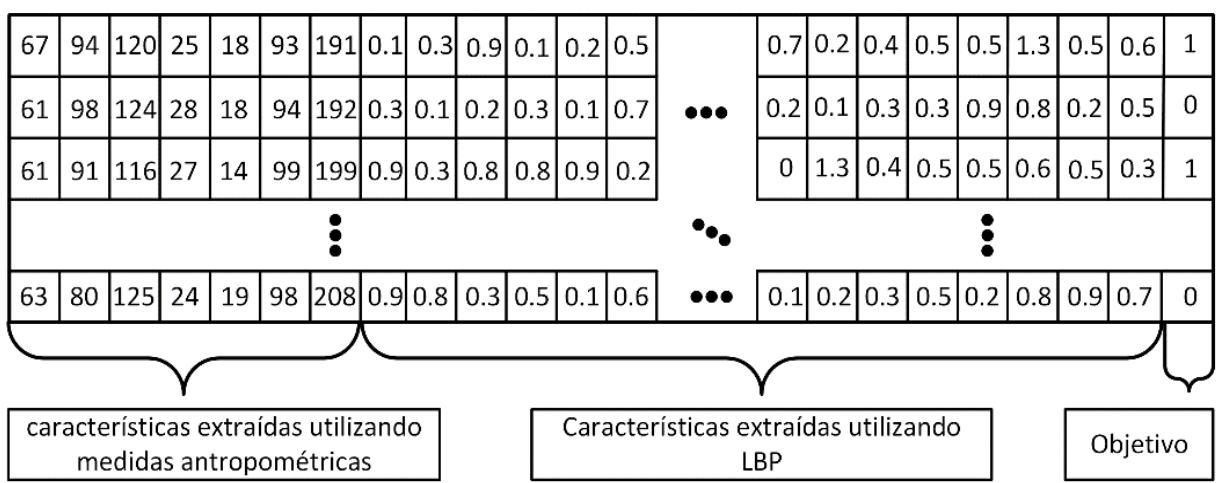

Fig. 11. Representación de la matriz de características.

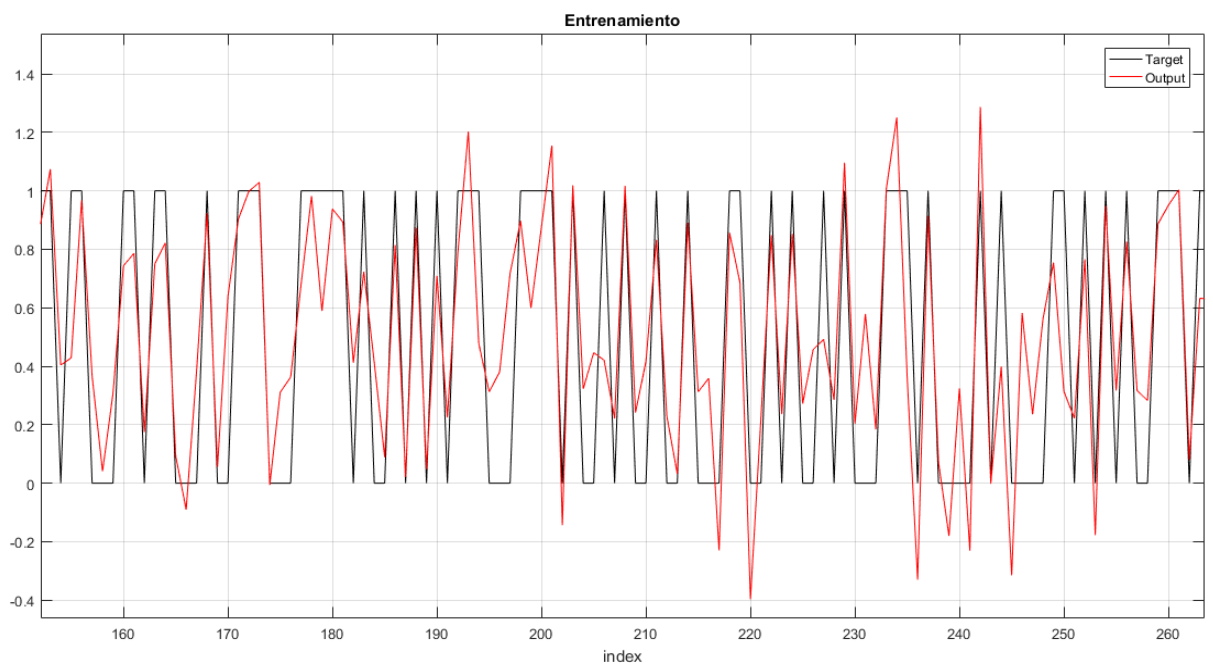

Fig. 12. Aproximación de ANFIS (línea roja) a los datos de entrenamiento (línea negra), se realizó un zoom al gráfico para hacer visible el comportamiento de la aproximación.

\section{Resultados}

A continuación se muestran los datos del entrenamiento de la arquitectura ANFIS: Se realizaron 100 epochs con una matriz de $1009 \times 66$ datos de entrenamiento y lo cual dio como resultado un MSE (Median Square Error) de 0.13551 y RMSE (Root Median Square Error) de 0.36812. En la Fig. 12 se muestra el comportamiento y la aproximación de los datos de entrenamiento conseguidos por ANFIS.

Para validar el modelo se utilizaron 428 imágenes distintas a las del entrenamiento, de las cuales 204 son de la clase femenina y 224 de la clase masculina. Estos datos fueron introducidos al modelo entrenado previamente. El porcentaje de precisión para 
cada clase se puede ver en la Tabla 2 y en la Fig. 13 la matriz de confusión, como se puede observar, la clase masculino demostró una mejor precisión. El promedio de clasificación total fue del $86.44 \%$.

Tabla 2. Rendimiento por clases utilizando la arquitectura ANFIS.

\begin{tabular}{cc}
\hline Clase & Porcentaje de clasificación \\
\hline Femenino & $83.33 \%$ \\
Masculino & $89.28 \%$ \\
\hline
\end{tabular}

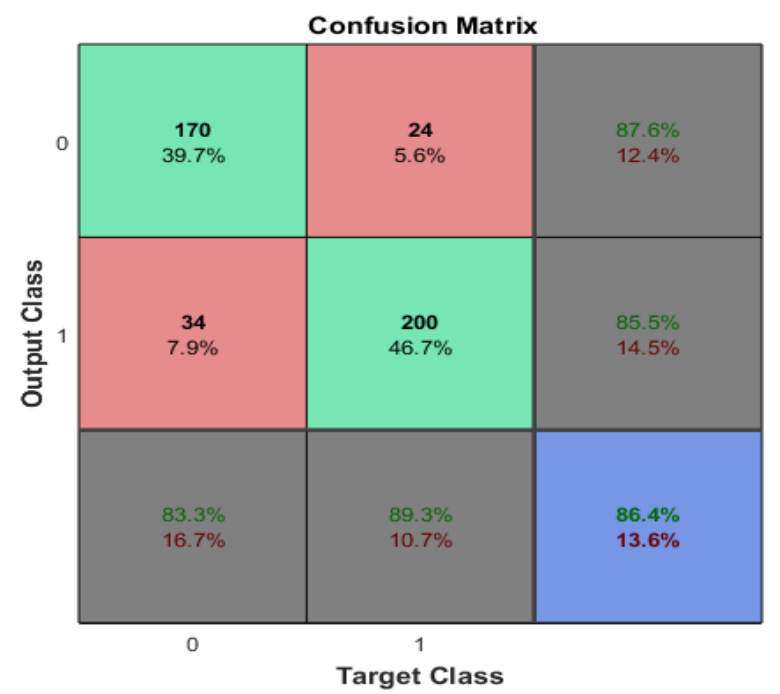

Fig. 13. Matriz de confusión que representa gráficamente los porcentajes de clasificación correcta e incorrecta de cada clase la clase 0 femenino y la clase 1 masculino.

En la tabla 3 se muestra la comparación del método propuesto con otros métodos, en la cual se puede observar un mejor desempeño de nuestro método. Las bases de datos utilizadas por los autores son las siguientes: [7] utilizó 42 imágenes para el entrenamiento, estas imágenes son obtenidas de la base de datos FERET, para realizar las pruebas los autores utilizaron 1199 imágenes de la misma base de datos. [15] utilizó las bases de datos FERET y FGNET, los autores utilizaron 1500 imágenes para realizar el entrenamiento y 200 imágenes para la realización de las pruebas, finalmente [12] utilizó la base de datos facial llamada Adience benchmark for age and gender classification, esta base de datos contiene 26000 imágenes, los autores utilizaron la versión que contiene los rostros ya recortados y alineados, el número de imágenes para el entrenamiento fue de 19487 y se utilizaron 6513 imágenes para las pruebas.

Cabe destacar que los autores [7] y [15] utilizaron imágenes en un entorno controlado como las usadas en esta investigación, y en [12] imágenes medianamente controladas, ya que estas son alineadas pero pueden contener ruido como: posturas no frontales y desenfoque en la imagen del rostro. 
Clasificación de género utilizando medidas antropométricas, análisis de texturas y la arquitectura ...

Tabla 3. Comparación del método propuesto con otros métodos.

\begin{tabular}{cc}
\hline Método & Porcentaje de clasificación \\
\hline P. Moallen y B. Mousavi [7] & $85.05 \%$ \\
T. Kalansuriya y A. Dharmaratne, [15] & $85.83 \%$ \\
G. Levi y T. Hassncer [12] & $86.35 \%$ \\
Método propuesto & $86.44 \%$ \\
\hline
\end{tabular}

\section{Conclusiones}

Un nuevo método para la clasificación de género es propuesto en este artículo basado en el análisis antropométrico y de texturas, en el que se consiguió un porcentaje de clasificación del $86.44 \%$. Se realizó el preprocesamiento de la imagen enfocado a la normalización del área del rostro sin importar el tamaño de la imagen, en la sección de extracción de características la combinación de las medidas antropométricas y LBP hasta el momento, han sido muy poco utilizadas específicamente en la clasificación de género. Las imágenes fueron clasificadas en dos grupos, masculino y femenino, la arquitectura ANFIS y el algoritmo FCM fueron utilizados para realizar la clasificación, los resultados experimentales demostraron que usando el algoritmo propuesto la clasificación de género se realiza con mayor o igual precisión que otros métodos.

\section{Referencias}

1. $\quad \mathrm{Fu}, \mathrm{S} ., \mathrm{He}, \mathrm{H} ., \mathrm{Hou}, \mathrm{Z}$. G.: Learning race from face: A survey. IEEE Trans. Pattern Anal. Mach. Intell., 36, pp. 2483-2509 (2014)

2. Colmenares-Guillen, L. E., Velasco-Albores, F. J., Moreno-Ledo, O. G.: Análisis de estudio de casos para estimar la edad basado en detección de rostros en las imágenes digitales [Case study analysis to estimate the age based on face detection digital images]. Int. J. Innov. Sci. Res., 25, pp. 130-140 (2016)

3. Ullah, I., Hussain, M., Muhammad, G., Aboalsamh, H., Bebis, G., Mirza, A. M.: Gender recognition from face images with local WLD descriptor, pp. 11-13 (2012)

4. Grother, P., Ngan, M.: Face Recognition Vendor Test ( FRVT ) Performance of Face Identification Algorithms. Natl. Inst. Stand. Technol. (2014)

5. Saravia, J. A.: Desarrollo de un método de clasificación de edad para imágenes faciales basado en análisis antropométrico y de texturas (2011)

6. Reid, D., Samangooei, S., Chen, C., Nixon, M., Ross, A.: Soft Biometrics for Surveillance : An Overview. Elsevier B.V. (2013)

7. Moallem, P., Mousavi, B. S.: Gender classification by fuzzy inference system. Int. J. Adv. Robot. Syst., 10 (2013)

8. Makinen, E., Raisamo, R.: Evaluation of Gender Classification Methods with Automatically Detected and Aligned Faces. IEEE Trans. Pattern Anal. Mach. Intell., 30, pp. 541-547 (2008)

9. Golomb, B. A., Lawrence, D. T., Sejnowski, T. J.: SexNet: A Neural Network Identifies Sex from Human Faces. In: Proceedings of the 1990 Conference on Advances in Neural Information Processing Systems, Morgan Kaufmann Publishers Inc., San Francisco, CA, 
USA, 3, pp. 572-577 (1990)

10. Otoole, A. J.: Sex classification is better with three-dimensional head structure than with image intensity information, 26 (1997)

11. Moghaddam, B., Ming-Hsuan, Y.: Learning Gender with Support Faces. IEEE Trans. Pattern Anal. Mach. Intell., 24 (2002)

12. Levi, G., Hassncer, T.: Age and gender classification using convolutional neural networks. 2015 IEEE Conf. Comput. Vis. Pattern Recognit. Workshop, pp. 34-42 (2015)

13. Baluja, S., Rowley, H. A.: Boosting Sex Identification Performance. Int. J. Comput. Vis., 71, pp. 111-119 (2007)

14. NIST: Face Recognition Technology. Disponible en: https://www.nist.gov/programsprojects/face-recognition-technology-feret.

15. Kalansuriya, T. R., Dharmaratne, A. T.: Neural Network based Age and Gender Classification for Facial Images. Int. J. Adv. ICT Emerg. Reg., 7, 1 (2015)

16. Lakra, S., Singh, J., Singh, A. K.: Automated pitch-based gender recognition using an adaptive neuro-fuzzy inference system. 2013 Int. Conf. Intell. Syst. Signal Process, pp. 82-86 (2013)

17. Sahu, S., Rao, A. P., Mishra, S. T.: Comparision between Neural Network And Adaptive Neuro- Fuzzy Inference System (Anfis) Results In Determination Of Gender Using Fingerprints, pp. 1064-1068 (2015)

18. Minear, M., Park, D. C.: A lifespan database of adult facial stimuli. Behav. Res. Methods. Instrum. Comput., 36, pp. 630-3 (2004)

19. Jang, J. S. R.: ANFIS: Adaptive-Network-Based Fuzzy Inference System. IEEE Trans. Syst. Man Cybern., 23, pp. 665-685 (1993)

20. Cortés, E., Algredo-Badillo, I., García, V. H.: Performance Analysis of ANFIS in short term Wind Speed Prediction. IJCSI Int. J. Comput. Sci. Issues, 9, pp. 9 (2012)

21. Viola, P., Jones, M. J.: Robust Real-Time Face Detection. Int. J. Comput. Vis., 57, pp. 137-154 (2004)

22. Dehshibi, M. M., Bastanfard, A.: A new algorithm for age recognition from facial images. Signal Processing, 90, pp. 2431-2444 (2010)

23. Amos, B., Ludwiczuk, B., Satyanarayanan, M.: OpenFace: A general-purpose face recognition library with mobile applications. (2016)

24. Pietikäinen, M., Hadid, A., Zhao, G., Ahonen, T.: Computer Vision Using Local Binary Patterns. Springer-Verlag London, London (2011)

25. Khanmohammadi, S., Ghaemi, S., Samadi, F.: Human Age Group Estimation Based on ANFIS Using HOG LBP Features. Electr. Electron. Eng. An Intenartional J., 2, pp. 2129 (2013)

26. Shan, C.: Learning local binary patterns for gender classification on real-world face images. Pattern Recognit. Lett., 33, pp. 431-437 (2012)

27. Navarro, R. I.: Study of a neural network-based system for stability augmentation of an airplane. (2013)

28. Brain, F. C. M., Segmentation, A., Ibrahim, S., Elaiza, N., Khalid, A., Manaf, M.: SeedBased Region Growing (SBRG) vs Adaptive Network-Based Inference System (ANFIS) vs Fuzzy, 4, pp. 315-325 (2010) 